\title{
Minimization of exergy losses in combustion processes with an illustration of a membrane combustion
}

\author{
Markku J. Lampinen*, Ralf Wiksten, Arto Sarvi, Kari Saari and Marjut Penttinen \\ Aalto University, Department of Energy Technology, Applied Thermodynamics, Sähkömiehentie 4J, P.O. Box \\ 14400, FI-00076 Aalto, Finland \\ *Corresponding author. Tel: +358-9-47023582, +358-500-448418, E-mail: Markku.Lampinen@tkk.fi
}

\begin{abstract}
The efficiency of internal combustion engines and gas turbine processes are free from Carnot limitations as they are not performing cycle processes - the initial state of the thermodynamic system is the fuel with air, whereas the final state is the flue gas, whose chemical composition is different than fuel and air. Therefore, as we show here, the theoretical thermodynamic efficiencies of ideal combustion engines and gas turbine processes can be very high, the same as it is for fuel cells. The entropy generation analysis, what we have done for the internal combustion engines and gas turbines, shows that they suffer for relatively low efficiencies because of the exergy losses in the combustion processes, i.e. for the reason that the combustion reaction takes place quite far from the equilibrium state. We have studied several different combustion processes in the Exergyproject of MIDE-program to find a method for decreasing the entropy generation in the combustion. If the entropy generation can be reduced, by any means, then as a "reward", we get the outlet pressure of the flue gas higher than the inlet air pressure without using any compressor which in turn would then increase the efficiency essentially. We present here a theoretical description of a membrane combustion method which, with the aid of gasification, suits for bioenergy. Shortly, it can be described as a molecular scale oxygen gas compressor driven by the combustion reaction, where the affecting force is amplified by the electric field across the membrane.
\end{abstract}

Keywords: Entropy, exergy, combustion, membrane, efficiency, combustion engine, gas turbine process

\section{Introduction}

In adiabatic combustion process the outlet temperature of the flue gas depends on the air factor $\lambda$ and the fuel. It does not depend on the pressure as far as we consider the flue gas as an ideal gas, because then the specific enthalpy of the species (i) depends only on its temperature: $h_{i}=h_{i}(T)$. In very high pressures near to the critical pressure, where the ideal gas assumption is no longer valid, the pressure affects also on the specific enthalpy.

Hence, the pressure of the outflow gas does not follow from the energy balance, but it depends on the manner how the combustion process is realized. So we need the second law of thermodynamics to analyze this. The specific entropy of the gas species (i) depends on the temperature $T$, and also on its partial pressure $p_{i}$, i.e. according to the ideal gas model $s_{i}\left(T, p_{i}\right)=s_{i}\left(T, p_{0}\right)-R \ln \left(p_{i} / p_{0}\right)$, which shows that the higher the pressure $p_{i}$ the smaller is the entropy $s_{i}$. Here the reference pressure $p_{0}=1$ bar and the gas constant $R=8.314 \mathrm{~J} / \mathrm{molK}$. The entropy generation in the adiabatic combustion is

$\sigma_{\text {irr }}=\sum_{\text {out }} n_{i} s_{i}-\sum_{\text {in }} n_{j} s_{j} \geq 0$

from which we see that the higher is the outlet pressure, the smaller is the entropy generation $\sigma_{i r r}$. Instead of the entropy generation we may as well speak of the exergy loss defined as

$T_{(-)} \sigma_{i r r}=$ exergy loss, 
which describes the loss of mechanical work in chemical combustion reaction, or the loss to increase the pressure of the flue gas by the chemical reaction. Temperature $T_{(-)}$is defined with the aid of the real final state $(B)$ and the ideal isentropic final state $\left(B_{s}\right)$ as follows [1]:

$T_{(-)} \equiv \frac{H\left(B_{s}\right)-H(B)}{S\left(B_{s}\right)-S(B)}$

Usually the flue gases from the combustion chambers in the gas turbine processes flow out approximately at the same pressure as the inlet flow of the air, and we speak then about combustion at constant pressure. In our earlier paper [2] we have shown that in the conventional combustion with constant pressure the entropy generation is very high and the exergy loss $\left(T_{(-)} \sigma_{i r r}\right)$, depending on the air factor and the fuel, is about half of the heat value of the fuel.

In the classical form of the Guoy-Stodola, the exergy loss is defined as $T_{o} \sigma_{i r r}$, where $T_{o}$ is the lowest temperature of the surroundings with which the system is in thermal contact. As we have shown in our earlier paper [2] the choice of the temperature $T_{o}$ for Eq.(2) does not give the accurate value for the lost of the work and for the efficiency of combustion engines (for Eq.(4) below), only an upper limit, and therefore, as shown in [2] we use the correct temperature $T_{(-)}$instead of $T_{o}$.

\section{Isentropic combustion}

The ideal adiabatic combustion process is such that there is no entropy generation, $\sigma_{i r r}=0$. In the language of thermodynamics it is called an isentropic combustion, and it means a combustion process which proceeds via equilibrium states. In the following we discuss how important for the efficiency it is to keep the entropy generation $\sigma_{i r r}$ as small as possible in order to have a good efficiency.

\subsection{Combustion engine}

For the combustion engine the following general equation for the efficiency can be derived [1]

$$
\eta=1-\frac{T_{(-)} \sigma_{i r r}}{-\left[H\left(B_{s}\right)-H(A)\right]}
$$

where $\sigma_{i r r}$ is the generation of entropy in the whole combustion engine process and $-\left[H\left(B_{s}\right)-H(A)\right]$ is the heat value of the isentropic combustion process from $A$ to $B_{s}$, which is defined so that $S(A)=S\left(B_{s}\right)$. In the denominator of Eq.(4) there is the enthalpy difference $-\left[H\left(B_{s}\right)-H(A)\right]$ because our system is a closed system which makes transformation process in the engine during $720^{\circ}$ degrees of rotation of the crank shaft. The entropy generation $\sigma_{i r r}(\mathrm{~J} / \mathrm{K})$ means correspondingly the entropy generation during $720^{\circ}$ degrees of rotation. 
We studied [1] the exergy losses of the diesel engine process, shown in Fig.1, and we found that $79 \%$ of all exergy losses took place between the combustion process steps 4-5-6-7. The whole efficiency of the diesel engine was $47.5 \%$. Therefore, if we could eliminate the exergy losses of combustion, then the exergy losses left were $21 \%$ x $52.5 \%=11 \%$ and the efficiency of the diesel engine would be $\eta=89 \%$. It can be so high as the theoretical efficiency can be even one as we see from Eq.(4). The reason for that is that the efficiency is not limited by Carnot formulae because the process is not a cycle process. The efficiency is under the same type of limitations as fuel cells, but for the combustion engine the reference process is an ideal reversible adiabatic process whereas for the fuel cells it is an ideal reversible isothermal process. Therefore, the maximum work out here is $-\Delta H(S=c o n s t)$, whereas for the fuel cell it is $-\Delta G(T=$ const, $p=$ const $)$.

How would then the ideal process without any exergy losses in the combustion steps look like compared to Figure 1a? First of all, the engine would be then a two stroke engine, but without having any dead volume. Suppose first that the piston is at left (with volume=0) and the hot flue gas starts to fill the cylinder by pushing the piston to the right in Fig.1b by constant pressure (say at 230 bar as in Fig.1a) from the point $4 *$ to the point $5^{*}$. At that point the inlet valve is closed and the isentropic expansion process starts, during which the pressure and the temperature become lower. The length of the piston stroke is such that the point $9^{*}$ is reached. After that the outlet valve is opened and the flue gas is flowing out to the turbocharger at constant pressure ( 3 bar in Figs.1a and 1b). All the flue gas is pushed off at constant pressure to the zero dead volume and then the process is repeated by filling the cylinder again by hot flue gas with a high pressure. The turbine unit of the turbocharger is rotating the shaft of the compressor which is feeding air into the special external membrane combustion chamber. That external membrane combustion chamber is assumed to produce the flue gas out at high pressure without any remarkable additional work (only the compression work for fuel feeding) and theoretically with zero entropy generation.

The efficiency of this type of a combustion engine shown Fig.1b is much higher than the engine in Fig.1 a because the compression work (3-4 in Fig.1a) is not needed and because the expansion (point 8) continues down to 3 bar whereas it stops now (Fig.1a) at 12 bar, which means that more expansion work can be taken out to the crank shaft by the process. 


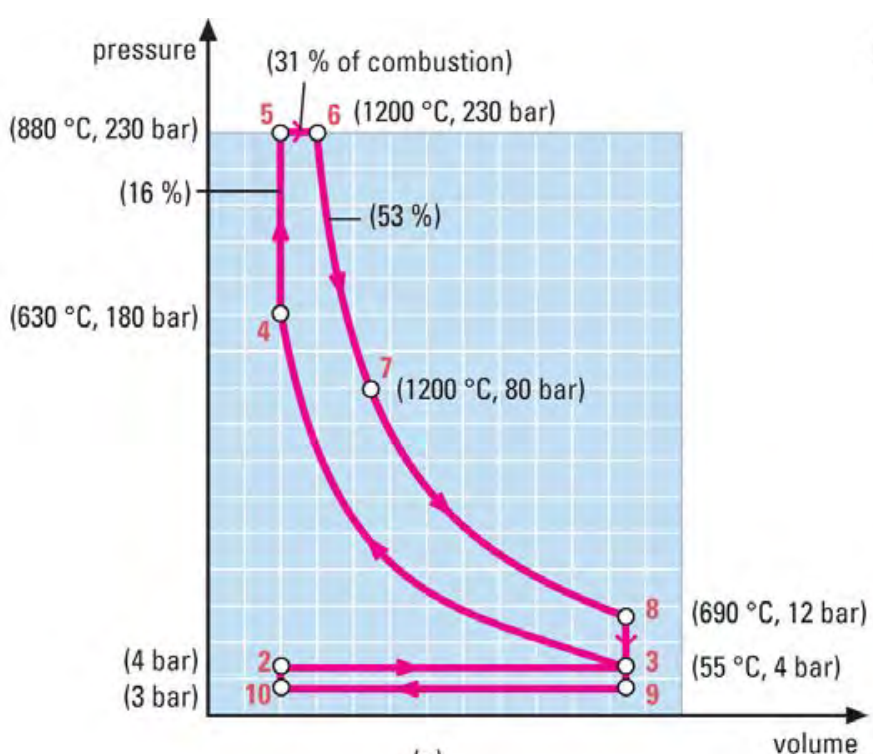

(a)

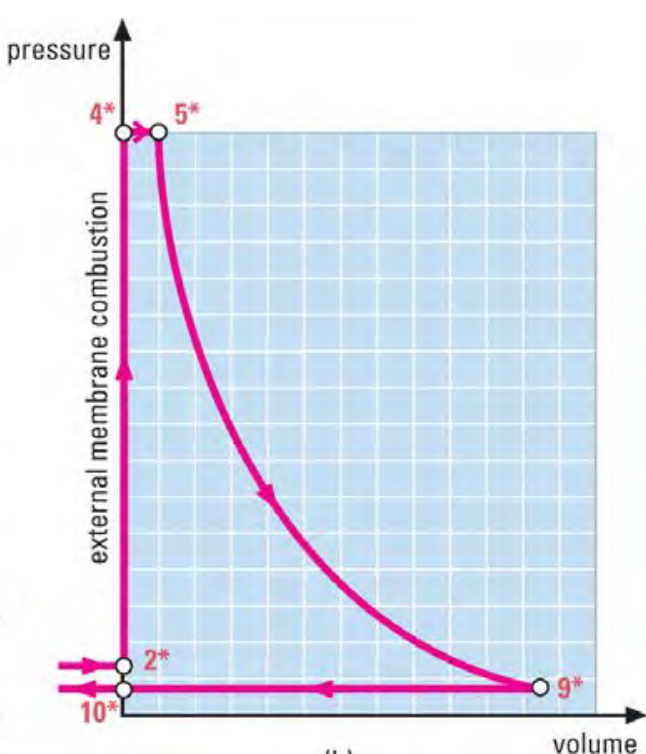

(b)

Fig. 1a. Turbocharged diesel engine process. Combustion takes place between 4-5-6-7.

1 b. Turbocharged external membrane combustion engine. Combustion only between $2 *-4 *$.

\subsection{Gas turbine process}

Also for the gas turbine processes the exergy losses in the combustion chamber are of crucial importance. In a methane gas driven power plant in Finland the gas turbine gives out $94 \mathrm{MW}$ of shaft power with the flue gas inlet at $1100{ }^{\circ} \mathrm{C}$ and pressure $11 \mathrm{bar}(\mathrm{abs})$. The compressor driven by the gas turbine takes $54 \mathrm{MW}$ which means that the shaft power delivered to the generator is $94 \mathrm{MW}-54 \mathrm{MW}=40 \mathrm{MW}$. By reducing sufficiently the entropy generation in the combustion unit the outlet pressure of $11 \mathrm{ba} \mathrm{r}$ could be achieved without using the compressor at all, and thus the whole turbine power could be transferred to the generator which of course, would increase the efficiency essentially. The principal power process based on the use of the theoretical isentropic combustion chamber is shown in Fig.2.

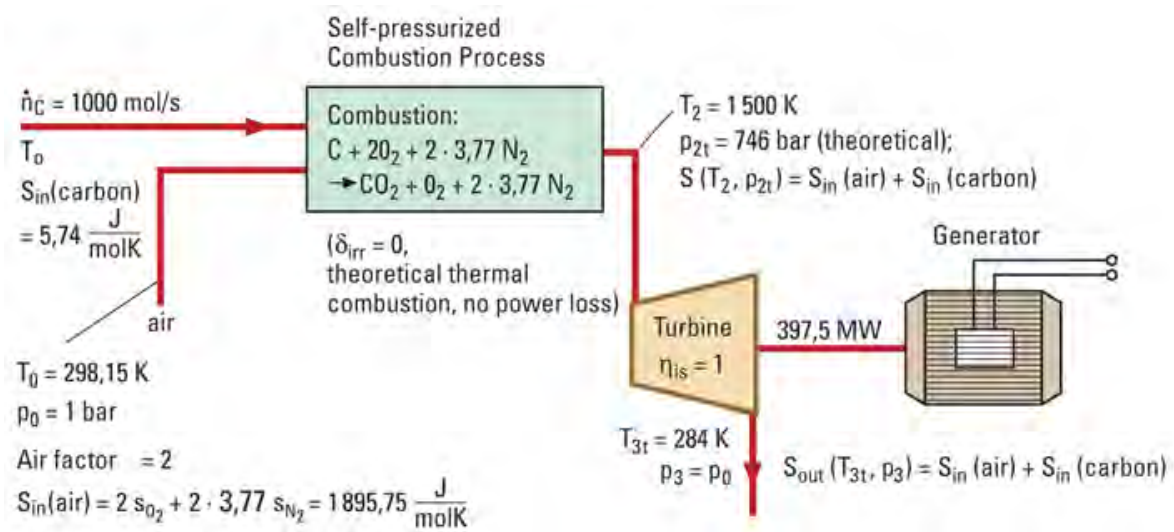

Fig. 2. Illustration of an ideal theoretical gas turbine process where the combustion takes place isentropically [1].

Figure 2 shows the theoretical limits for the gas turbine process if the entropy generation is zero in the combustion chamber and also in the turbine. If $\sigma_{i r r}=0$ in the combustion chamber, then as shown in Fig.2, the outlet pressure will be as high as $p_{\text {out }}=746$ bar. This is, of course just a theoretical number, but it shows that there is a great potential to improve the conventional combustion process. For instance, to achieve pressure ratio $p_{\text {out }} / p_{\text {in }}=11$ in the 
combustion chamber with carbon as fuel and with $\lambda=1$, we need to decrease entropy generation from $\sigma_{i r r}=366 \mathrm{~J} / \mathrm{molK}$ only to $\sigma_{i r r}=270 \mathrm{~J} / \mathrm{molK}$. In the following we will shortly discuss how we could do this by a membrane combustion.

\section{Principle of semipermeable membrane combustion}

Ceramic membranes made of yttria-stabilized zirconia (in Fig. 3: $\mathrm{ZrO}_{2} / \mathrm{Y}_{2} \mathrm{O}_{3}$ ) has the property that in sufficient high temperatures $\left(800-1000{ }^{\circ} \mathrm{C}\right)$ they start to conduct oxygen ions $\left(\mathrm{O}^{2-}\right)$. The ionic conductivity depends on the amount of yttria in the structure. Approximately half of the amount of yttria atoms in the crystalline structure can offer vacancies to be occupied by "hopping" oxygen ions. These materials are well known from Solid Oxide Fuel Cells and from lamda-sensors used in cars for measuring the oxygen concentration in flue gases.

As shown in Fig.3, the oxygen gas is consumed on the surface of the combustion side and therefore, the partial pressure of oxygen gas becomes there lower than on the surface of the airside. Because of the ionic form of oxygen, the difference of partial pressures generates a potential difference, which can be estimated by Nernst equation (see Fig.3). The electric field performed by the potential difference is the driving force for the oxygen transport through the membrane. Depending on the concentration of oxygen ions, the electric volumetric force field (ion charge density $\mathrm{x}$ electric field, $\mathrm{N} / \mathrm{m}^{3}$ ) can be amplified to several magnitudes higher than the partial pressure gradient of oxygen gas.

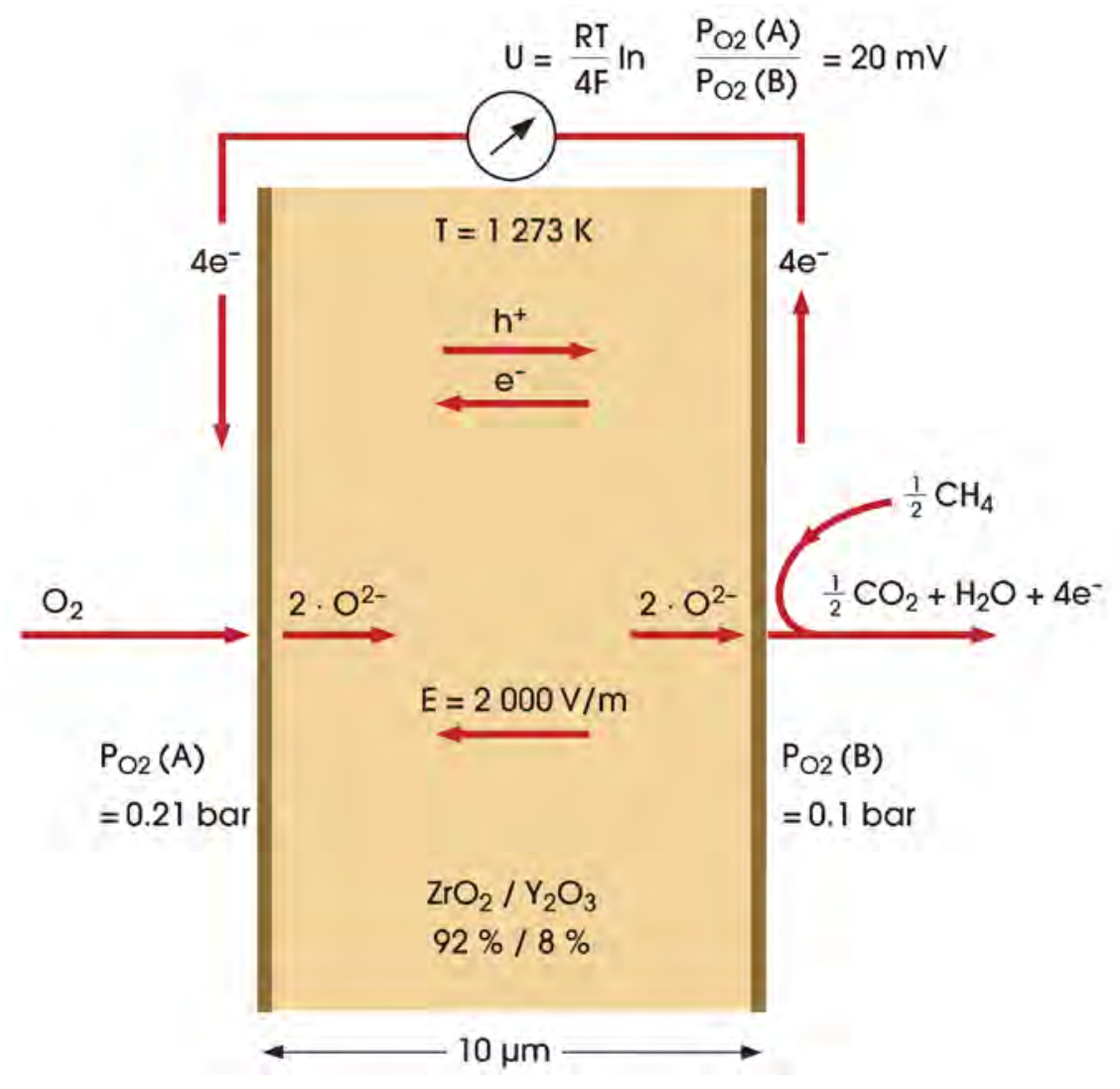

Fig. 3. Semipermeable oxygen gas membrane. Electric potential difference across the membrane is illustrated by assuming that the partial pressures of oxygen are 0.21 bar (on the air side) and 0.1 bar (on the flue gas side). The total pressure of the flue gas $p(B)$ is kept higher than the total pressure of air $p(A)$ by adjusting the outlet flow of flue gas accordingly. 
Recently, it has been studied the ionic and electronic charge transport for single crystals of yttria-stabilized zirconia with additional nitrogen doping, e.g. [3] and [4]. At temperatures above $850{ }^{\circ} \mathrm{C}$, even in the presence of a very small oxygen concentration in the surrounding gas phase, the nitrogen ion dopant becomes highly mobile, and thus diffuses to the surface where it is oxidized to gaseous $\mathrm{N}_{2}(\mathrm{~g})$. The technical motivation for that study [3] has been to achieve sufficient nitrogen ion conductivity for the development of a nitrogen sensor or nitrogen pumps. In the membrane combustion the driving force for the nitrogen transportation comes also from the electric field generated by the oxidation of the fuel. The flow of nitrogen gas lowers the temperature of the membrane, which merely by oxygen combustion would be too high. A construction using nitrogen and oxygen gas semipermeable membranes is shown in Fig.4.

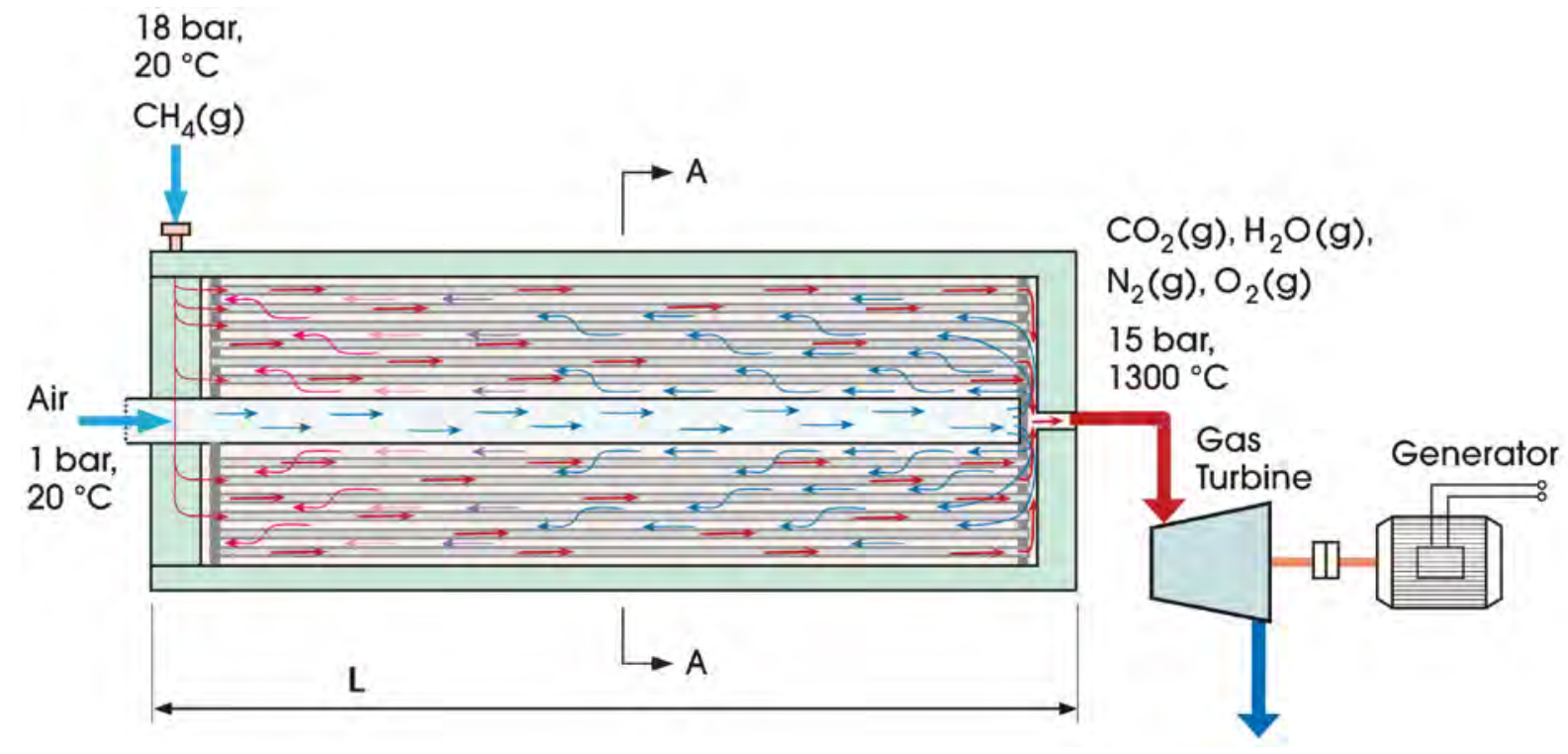

Fig. 4. Hollow ceramic fibers used in the membrane combustion. Fuel (here the methane gas) is fed in the fibers and air outside of the fibers.

\section{Discussion}

We have discussed here that the entropy generation in the combustion processes is the crucial point that reduces the efficiencies of combustion engines and gas turbine processes. We have presented here an illustration of a membrane combustion method which, with the aid gasification is quite suitable for bioenergy, and which can reduce the entropy generation by pressurizing the combustion chamber without using any external work. Shortly, it can be described as a molecular scale gas compressor driven by the combustion reaction, where the affecting force is amplified by the electric field across the membrane.

\section{Acknowledgements}

This study was supported and financed by the MIDE-program of Aalto University. We wish to thank prof. Yrjö Neuvo and Dr. Sami Ylönen for the kind support to this work. The authors wish to acknowledge also prof. Frank Pettersson and prof. Ron Zevenhoven from Åbo Akademi for many useful discussions. 


\section{References}

[1] M.J. Lampinen, R. Wiksten, A. Sarvi, K. Saari and M. Penttinen: Minimization of exergy losses in combustion processes and its consequences. XXII Sitges Conference on Statistical Mechanics. Energy Conversion: From Nanomachines to Renewable Sources. Sitges, Barcelona, Spain, 7-11, June 2010.

[2] M.J. Lampinen and R. Wiksten, Theory of effective heat-absorbing and heat-emitting temperatures in entropy and exergy analysis with applications to flow systems and combustion processes. Journal of Non-Equilibrium Thermodynamics 31, 2006, pp. 257-291.

[3] I. Valov, V. Ruhrup, R. Klein, T.-C. Rödel, A. Stork, S. Berendts, M. Dogan, H.-D. Wiemhöfer, M. Lerch, J. Janek, Ionic and electronic conductivity of nitrogen-doped YSZ single crystals. Solid State Ionics 180, 2009, pp. 1463-1470.

[4] M. Lerch, J. Janek, K.D. Becker, S. Brendts, H. Boysen, T. Bredow, R. Dronskowski, S.G. Ebbinghaus, M. Kilo, M.W. Lumey, M. Martin, C. Reimann, E. Schweda, I. Valov, H.D., Wiemhöfer, Oxide nitrides: From oxides to solids with mobile nitrogen ions Progress in Solid State Chemistry 37, 2009, pp. 81-131. 